\title{
Blueprint for the Development and Sustainability of National Nanosafety Centers
}

\author{
Neeraj Shandilya (1D - Effie Marcoulaki • Sven Vercauteren • Hilda Witters • \\ Eric Johansson Salazar-Sandoval • Anna-Kaisa Viitanen • Christophe Bressot • \\ Wouter Fransman
}

Received: 18 September 2019 / Accepted: 10 February 2020 / Published online: 19 March 2020

(C) The Author(s) 2020

\begin{abstract}
This work presents a blueprint or set of guidelines for the planning and development of sustainable national centers dealing with the safety of nanomaterials and nanotechnologies toward public health and environment. The blueprint was developed following a methodological approach of EU-wide online survey and workshop with several stakeholders. The purpose was to identify the key elements and challenges in the development and sustainability of a national nanosafety center. The responses were received from representatives of 16 national nanosafety centers across Europe and 44 people from 18 EU member states who
\end{abstract}

Electronic supplementary material The online version of this article (https://doi.org/10.1007/s11569-020-00364-6 ) contains supplementary material, which is available to authorized users.

N. Shandilya $(\bowtie) \cdot$ W. Fransman

TNO, Utrechtseweg 48, 3704 HE Zeist, Netherlands

e-mail: neeraj.shandilya@tno.nl

W. Fransman

e-mail: wouter.fransman@tno.nl

E. Marcoulaki

National Center for Scientific Research "Demokritos", Patr. Grigoriou E' \& Neapoleos 27, 15341 Agia Paraskevi, Greece e-mail: emarcoulaki@ipta.demokritos.gr

S. Vercauteren $\cdot H$. Witters

Department Health, VITO, Boeretang 200, 2400 Mol, Belgium

S. Vercauteren

e-mail: sven.vercauteren@vito.be represented the stakeholder groups of researchers, academics, industry, regulators, civil society, and consultants. By providing an overview of the organizational design of existing national nanosafety centers across EU and converging demands in the field of nanosafety, the blueprint principally benefits those EU member states who do not have a national nanosafety center, but intend to develop an entity to manage the human health, environmental, ethical, and social concerns/risks toward the growing nationwide activities on engineered nanomaterials, e.g., their production, use or disposal, at national level.
H. Witters

e-mail: hilda.witters@vito.be

E. J. Salazar-Sandoval

RISE Research Institutes of Sweden, Box 5607, SE-114

86 Stockholm, Sweden

e-mail: eric.johanssonsalazar-sandoval@ri.se

\author{
A.-K. Viitanen \\ Finnish Institute of Occupational Health, PO Box 40, \\ FI-00032 Työterveyslaitos, Finland \\ e-mail: anna-kaisa.viitanen@ttl.fi \\ C. Bressot \\ DRC/CARA/PROX, INERIS, BP 2, 60550 Verneuil en Halatte, \\ France \\ e-mail: christophe.bressot@ineris.fr
}


Keywords Engineered nanomaterials · Organizational design $\cdot$ Sustainability $\cdot$ Nanosafety center $\cdot$ Stakeholder concerns

\section{Introduction}

Nanotechnology has been identified as one of the key enabling technologies in the Horizon 2020 (H2020) Programme of the European Union (EU). It, thus, underlines the significance and benefit of this field for EU competitiveness and its ability to provide the innovative goods and services essential for meeting global challenges [1-3]. For the development of nanotechnology along the innovation chain, engineered nanomaterials (ENMs), nano-enabled products, and applications need a clear and easy-to-follow human and environmental safety framework [4]. A significant challenge to such a framework is to understand safety and health risks of the technology and its end products and to implement practical strategies to manage these risks [5-9]. Examining these risks and benefit perceptions utilized in the formation of attitudes and opinions about nanotechnology can be useful for both industry and policy makers involved in its development, implementation, and regulation [10]. To address these concerns, considerable effort has already been undertaken by the OECD Working Party on Manufactured Nanomaterials (WPMN) as well as the EU Framework Programmes FP6, FP7, and H2020 to answer basic scientific, technical, and social questions [11-13]. Nevertheless, knowledge is growing rapidly and effective use of this knowledge for risk management is lagging behind [11]. We therefore need to bridge the gap between knowledge on hazard and risk and fit-forpurpose risk management tools and strategies supported by measurement and control methods [14].

The national nanosafety centers play a pivoting role to bridge this gap at their respective national levels. A National Nanosafety Center (NNC) is herein defined as a national level entity that offers a broad range of services on the safe production, handling, and/or disposal of ENMs or nano-enabled products while strengthening the communication and cooperation between different stakeholders (e.g., consumer, industry, research organizations, and government) to ensure knowledge exchange in the field of environmental and human health safety toward ENMs (i.e., nanosafety). An overview, to the best of our knowledge, of various existing NNCs is provided in Table 1. The scope of operations and services of these NNCs vary from one to another, as presented later in this study. There are numerous other nanosafety centers too which operate on regional levels within a particular EU member state like the Namur NanoSafety Centre in Belgium, the Groupement d'Intérêt Scientifique (GIS) in France, NanoLab in Italy, EHS-Advance in Spain, NanoSafety in Austria, and Cluster Nanotechnology, NanoMat, Leibniz Research Alliance Nanosafety and NanoNetzwerkHessen in Germany. Such centers are either initiated by respective state/regional governments or run for a definite period of time. However, we limit the scope of this study to the centers operating at national levels.

As can be seen from Table 1, most of the EU member states are missing from it, and the majority of these member states are concentrated in the eastern EU. This does not necessarily imply that they are not involved in the production, use, or disposal of ENMs nor that they have no need to assess the safety of ENMs and its social implications. In fact, the European Commission recently reported a preliminary estimate of annual production quantities for 12 selected ENMs to exceed one million tons and their environmental release quantities to be approx. 3350 tons, distributed all over EU [15]. Within the framework of the present study, we observed that despite the ongoing investments to build, staff, and operate the nanosafety management in the member states which lack an NNC, the activities are mainly at the fundamental research level, e.g., in university laboratories, which lack inclusive collaboration network and dynamic dialog between different stakeholders at both local and national levels. In agreement with the Closerto-the-market (CTTM) Roadmap of the European Nanosafety Cluster (EU-NSC) [4], the development of the new NNCs in these member states will not only instill collaboration and dialog but would also facilitate market implementation of nano-enabled products, services, behaviors, or technology at local or national level commerce in them.

In 2016, an EU Horizon 2020 project was launched to initiate the setup of a European Center for Risk Management and Safe Innovation in Nanomaterials (EC4SafeNano) [16]. One of the primary objectives of the soon-to-be-established EC4SafeNano will be to serve as an umbrella organization for coordinating and networking with existing NNCs and stimulate the development of new NNCs. The present study, being a part of EC4SafeNano and the first of its kind, focuses on this objective by presenting a blueprint which aims to 


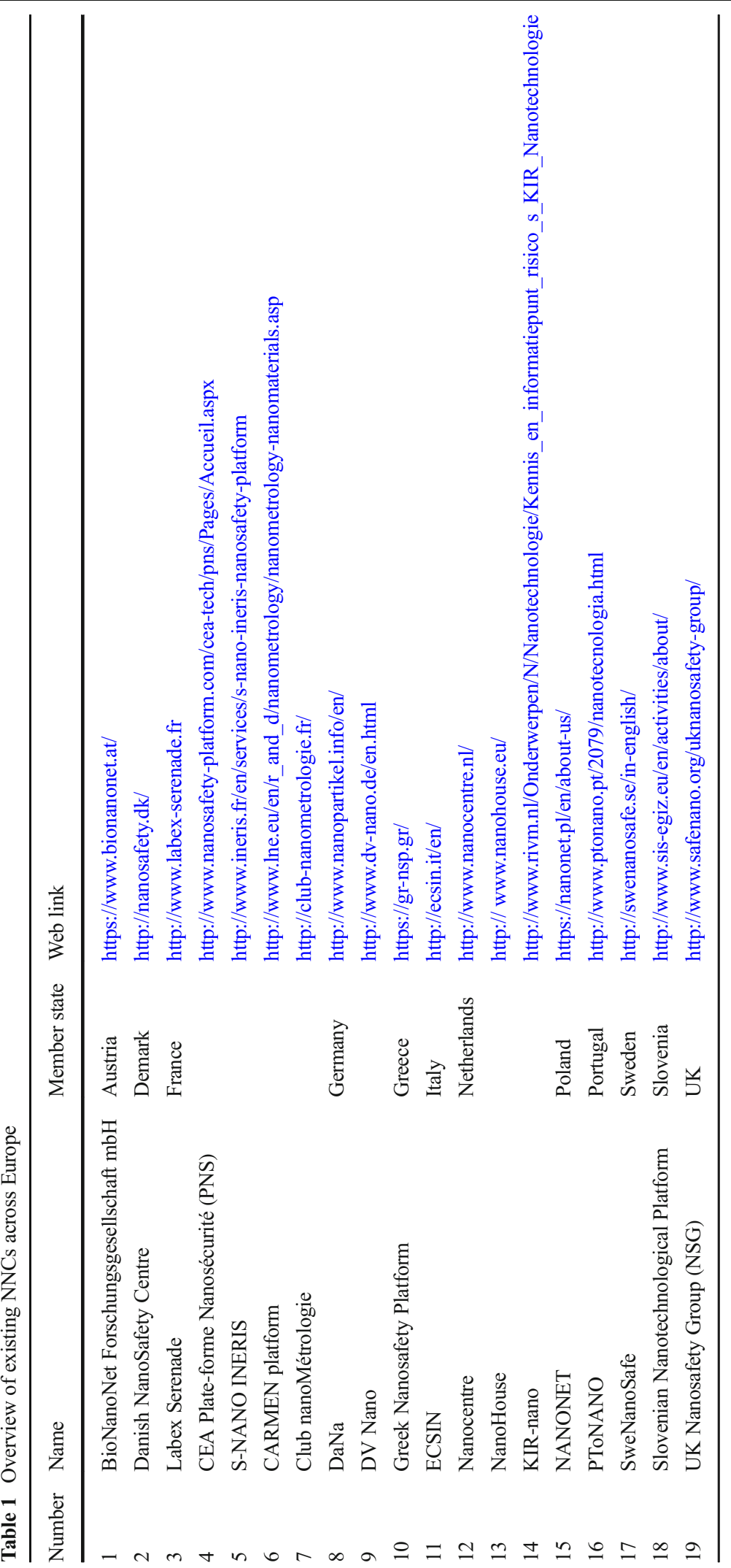


provide information or guidelines on the key elements which are vital in the development and sustainability of the NNC. The blueprint was developed on the basis of the information collected during an online survey of the existing NNCs and a workshop with different relevant stakeholder groups. While the blueprint gives an overview of the organizational design of existing NNCs across the EU and converging demands in the field of nanosafety, it principally benefits those EU member states that do not have an NNC (e.g., countries missing from Table 1) but desire to develop one to manage the human health, environmental, ethical, and social concerns/risks in their countries toward ENMs.

\section{Methodology}

\section{Online Survey}

In the first phase of the blueprint development, an online survey of the 19 NNCs in Table 1 was carried out from September 11 to October 13, 2018. The survey was carried out online using a questionnaire which was constructed to collect the information about the organization model, objectives, services, network, communication, funding, and clientele of an NNC. Before it was published online, a first draft of the questionnaire was circulated among the EC4SafeNano project consortium partners for their review. They were also briefed about the purpose and content of the questionnaire. The reviewing partners are the national expert institutes/ organizations which already provide expert support and services to the industry and other private actors, public authorities, and regulatory bodies. Their contact details can be found on the EC4SafeNano website (http://www.ec4safenano.eu/project-partners). In addition, three of the partners are also associated/involved with three NNCs in three different countries. The development of the draft questionnaire was based on various sources which included websites and published documents from NNCs in Table 1, EUNSC publications, and documents delivered within the framework of EC4SafeNano project. The questionnaire was edited according to the received feedback, and the final version was published online (provided in supplementary information Appendix A). The representatives from 19 NNCs in Table 1 were invited to respond to the survey and 16 respondents that completed the survey represented 16 different NNCs.
Workshop

The second phase consisted of an interactive workshop which was organized on November 5, 2018 during the Sixth International Conference nanoSAFE 2018 in Grenoble, France [17]. In total, 44 people participated in this workshop from $18 \mathrm{EU}$ member states, i.e., Austria, Belgium, Bulgaria, Denmark, Finland, France, Germany, Greece, Ireland, Italy, Luxembourg, Netherlands, Poland, Portugal, Spain, Sweden, Switzerland, and UK. The group of participants are comprised of various stakeholder groups of researchers (13), academics (12), industry (9), civil society (3), regulators (4), consultants (2), and government (EC; 1). They were asked to vote on the list of questions presented in supplementary information Appendix B. The questions mainly focused on their preferences, concerns, and needs while interacting with the NNCs. The voting was done using an interactive tool (Mentimeter, https://www. mentimeter.com/) in which the participants could see the number of received votes for each option in realtime. This enabled lively discussion among the stakeholder groups on the basis of their opinions on the received votes.

\section{Results}

The key elements for the development and sustainability of an NNC can vary from one organization type to another. Nevertheless, its structure, network, and clientele consistently determine the underlying dimensions of its operation model [18-20], thus serve as fundamental elements for an NNC to consider, along with its intended objective(s) and service(s), in translating its strategic intent into operational capabilities. For a successful operation model, a center with efficient, flexible, and innovative organizational structure should work in an engaging, extensive, and efficient network to provide the services to the clientele at the most satisfactory level. The results from the online survey and the workshop are described in the following sections. The terminology used in the text is defined in supplementary information Appendix C.

\section{Organization Structure of NNCs}

Figure $1 \mathrm{a}$ and $\mathrm{b}$ presents the responses for the concurrent types and structures of the organization, respectively, 
Fig. 1 Percentage of NNCs with different a organization types and b structures
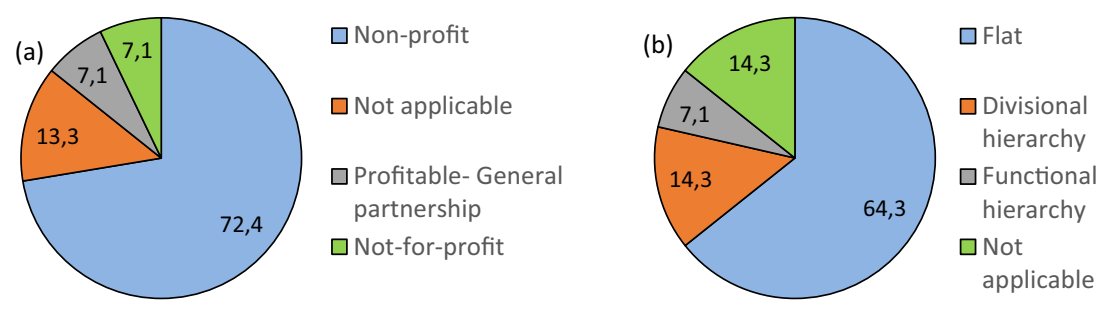

which are practiced by the existing NNCs. The large majority of the responses favor a non-profit type (72\% approx.) legal entity and flat organization (64\% approx.) structure of the center in which there are no middle management levels between governance and staff roles. A minor faction (approx. 13\%) of NNCs prefers to have no legal status (not applicable in Fig. 1a). According to Fig. 1b, most of the participating NNCs (64\%) are based on a flat hierarchy, followed by $14 \%$ using a divisional hierarchy system, and another $14 \%$ which do not follow any kind of organization type (i.e., not applicable).

Public funding is common for all NNCs as the most realistic form of sustainable revenue source. Almost half of the NNC also have an additional private component of their funding through their clients (see Fig. 2a). As shown in Fig. 2b, approx. 73-78\% of the NNCs have only a couple of people as head, advisory board, and management, to ensure sustainable operation, and over ten people are involved in multidisciplinary scientific research, to ensure better scientific relevance of the
NNCs regarding nanosafety. In terms of administration, public relations, and marketing personnel, they usually have one or two people (in approx. $50-57 \%$ of the NNCs), followed by none (approx. $43 \%$ for administration and marketing; approx. 29\% for public relations).

\section{Objective(s) of NNCs}

Based on their responses, each of the NNCs aimed toward multiple objectives which are considered by NNCs to be most relevant, strategic, and operational in the contemporary realm of nanosafety. The responses are shown in Fig. 3.

1. The most common objective is to create a network in the field of nanotechnologies and to establish a bridge between the industrial and academic worlds (approx. 86\%).

2. Approximately $71 \%$ of the respondents are interested in the initiation and coordination (or management) of
Fig. 2 Percentage of NNCs with different a funding or revenue sources and $\mathbf{b}$ personnel organization
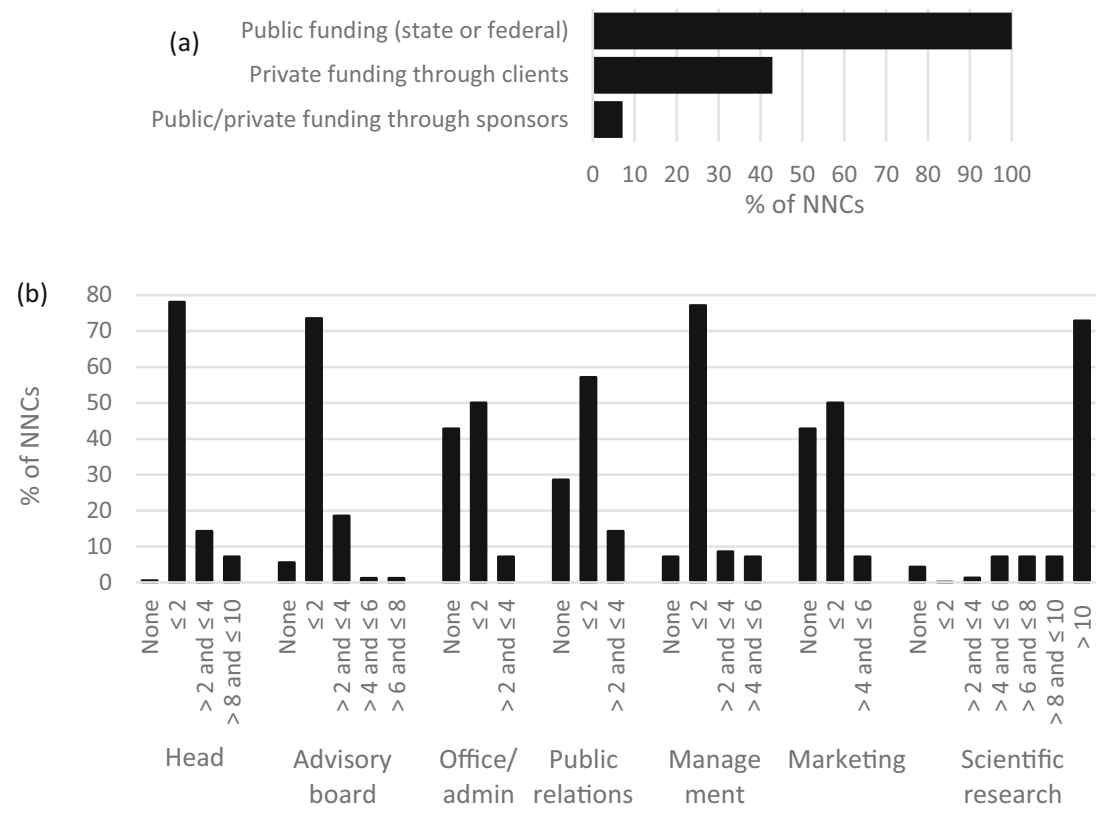
Fig. 3 Percentage of NNCs with different objectives

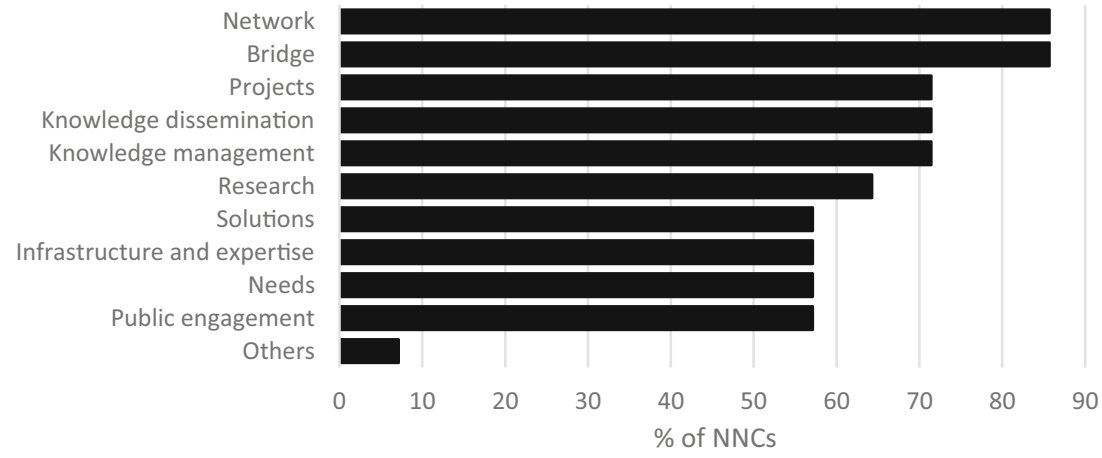

nanosafety related funded projects; the dissemination of knowledge to the industrial, academic and political stakeholders, and civil society through guides, technical procedures, press, media etc.; and the establishment and implementation of knowledge management in nanosafety, nano-ethics, and nano-enabled products.

3. Approx. $64 \%$ of NNCs aim to support innovative research for the safer application and implication of nanotechnology as well as to provide solutions and advices to the industries, laboratories, and regulatory agencies on exposure, toxicological, and ecotoxicological studies of nanomaterials.

4. Providing solutions, access to the infrastructure, and expertise in the network of an NNC are prioritized by $57 \%$ of the NNCs. An equal percentage of NNCs collect and specify the needs in risk assessment as well as strengthen and promote public engagement activities through various outreach programs and public forums.

Other objectives, such as (i) development of strategic documents and research programs, (ii) support toward standardization work and new businesses, and (iii) lobbying were observed to be prioritized by totally $7 \%$ of NNCs.

\section{Service(s) of NNCs}

To attain the aforementioned objectives in a financial sustainable manner, the NNCs provide multiple services through their networks. As shown in Fig. 4a, these services can be overall classified in five categories: (i) support services which are most popular and provided by approx. $84 \%$ of NNCs, (ii) training services in which $67 \%$ of the NNCs are interested, (iii) testing and analysis services are provided by $54 \%$ of the NNCs, (iv) a similar percentage $(50 \%)$ of the NNCs provide consultancy services, and (v) conformity assessment and certification services provided by less than $10 \%$ of the NNCs. When these percentages are compared with the response of the stakeholders (other than NNCs) regarding the services which they prefer to have or demand (Fig. 4a), we observe a match between service supply from NNCs and demand from other stakeholders, except for conformity assessment and certification services in which huge demands (from $64 \%$ of stakeholders) for little provided services (from approx. $9 \%$ of the NNCs) can be seen. Approx. $67 \%$ of the NNCs providing the support services do so by publishing newsletters (Fig. 4b). In addition to the newsletters, approx. 44\% of these NNCs also have a helpdesk and respond to frequently asked questions (FAQs) from different stakeholders as well as technological surveillance of nanotechnology and nanomaterials. Other support services include publishing whitepapers, sector guidance, and organizing workshops (each with a share of approx. $11 \%$ ). One should note that these support services are of particular interest to the stakeholder group of civil society (as observed during the workshop discussion) which emphasize on understanding and prevention of possible risks of nanomaterials as their primary concern regarding nanosafety.

In the context of training services (Fig. 4c), approx. $71 \%$ of the NNCs prefer to provide general training on Environmental Health and Safety (EHS) aspects. Tailormade or customized training is also provided by these NNCs but with lower fraction (approx. 37\%). Other less important training services for the NNCs include teaching activities and characterization of ENMs (approx. $14 \%$ each).

Physiochemical characterization of ENMs is provided by all NNCs which are busy in testing and analysis 


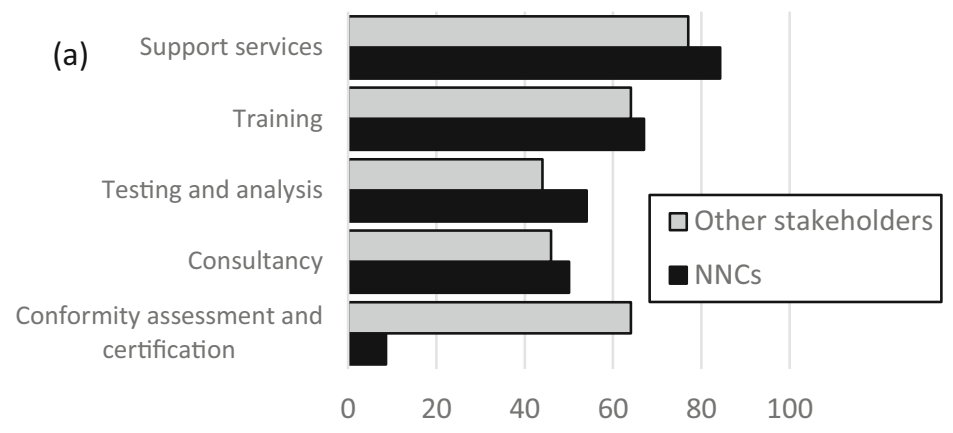

Response \%

(b)

$$
\begin{aligned}
& \text { Newsletters } \\
& \text { Helpdesk \& FAQ }
\end{aligned}
$$

Technological surveillance

Whitepapers

Sector guidance

Workshops

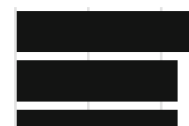

\section{.}

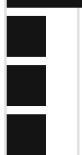

0

(c) General Training

Tailor made training

Teaching activities

Physio-chemical characterization
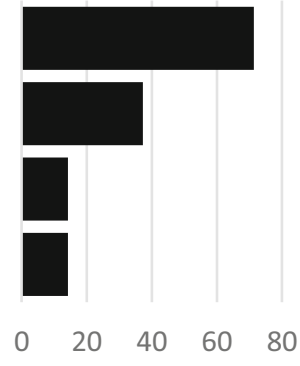

$\%$ of NNCS

(e) Risk analysis and assessment

Development of methods and

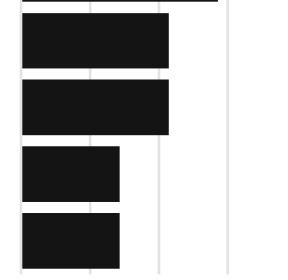

Exposure or release assessment

ENM transfer

Efficiency Assessment

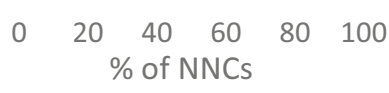
tools

Implementation of management systems

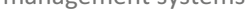

Regulatory
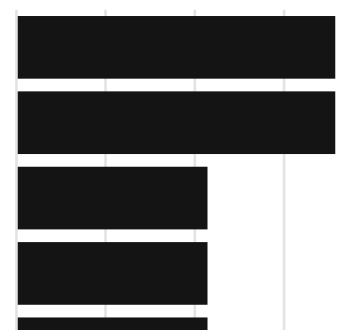

Safety procedures

Market sector

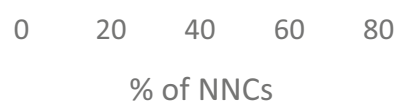

(f) Standardization and preCalibrations and metrology

Certification

Conformity Assessment

Voluntary Marks or technical conformity

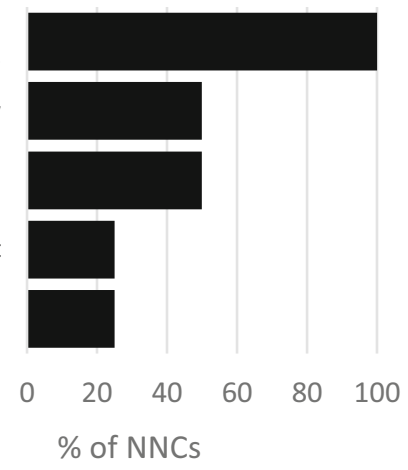

Fig. 4 a Classification of services, percentage of NNCs providing them, and comparison with the demand from other stakeholders. Percentage of NNCs providing different types of $\mathbf{b}$ support

services, $\mathbf{c}$ training services, $\mathbf{d}$ testing and analysis services, e consultancy services, and $\mathbf{f}$ conformity assessment and certification services 
(Fig. 4d). Approx. 71\% of the NNCs provide toxicology and 57\% aging test services. The other testing and analysis services include environmental fate and ecotoxicity (approx. 43\%), exposure or release assessment (approx. 43\%), ENM transfer (approx. 29\%), and efficiency assessment (approx. 29\%).

In terms of consultancy services, Fig. $4 \mathrm{e}$ shows that the risk analysis and assessment of ENMs and development of methods and tools are the most highly provided consultancy services (by approx. $71 \%$ of the NNCs). Approx. $43 \%$ of the NNCs prefer to provide services in the implementation of risk management systems, regulatory services, and safety procedures. Market sector consultancy is also one of the services provide by the NNCs but to a lesser extent (approx. 29\%).

The NNCs providing services in conformity assessment and certification are already limited (less than $10 \%$ ), and the NNCs which do provide this service prefer to perform standardization and prestandardization activities (Fig. 4f). Instrumental calibrations and metrology and different certification services are also provided by half of them. For the aforementioned supply-demand mismatch in the case of these services, there can be numerous possible reasons to which such a mismatch can be attributed. For instance, the existing NNCs could have failed to identify that conformity assessment and certification services have a strong demand among other stakeholders. Another reason can be that the stakeholders do not approach existing NNCs for such services but rather other entities, for example, in-house laboratories, stand-alone academics or collaborative entities (between research institutes or universities), because of their financial or other operational issues. Existing regulations concerned with nanomaterials (such as REACH) can be another reason why nanomaterials were not considered separately from other conventional chemical substances until January 2020 [21]. There has also been a lack of certificates and guidance documents on nanomaterials [22], which led to the creation of the Malta Initiative in 2018-2019 (https://www. nanosafetycluster.eu/international-cooperation/themalta-initiative/). The high demand for conformity assessment and certification for nanomaterials and nano-enabled products was being fulfilled by the aforementioned entities.

\section{Knowledge Dissemination and Management in NNCs}

As presented in section 3.2, knowledge dissemination was identified among the objectives of over $70 \%$ of the NNCs to tackle the ethical and social concerns surrounding ENMs. These NNCs were observed to be selective to disseminate and to manage their knowledge among different stakeholders as over half of them (approx. 57\%) choose to be open about information sharing (Fig. 5a). Figure 5b shows that $91 \%$ of these NNCs choose different meetings, forums or events (e.g., conferences, symposia, etc.), and newsletters as means for the dissemination and management of their nanosafety- and nanotechnology-relevant knowledge among the stakeholders. Social media is also chosen as an additional tool by approx. 35\% of them. A minor fraction of them (approx. 2\%) also consider other tools like webinars, word-to-mouth, internet blogs, articles, etc. to further disseminate the knowledge. A similar trend was observed among stakeholders participating in the workshop when they were asked to identify the most frequent ways through which they gather their knowledge about new developments in nanosafety. We thus observed a match between the means through which the NNCs disseminate their knowledge and other stakeholders gather their knowledge. Back to Fig. $5 \mathrm{a}$, out of the remaining $43 \%$ of the (a)

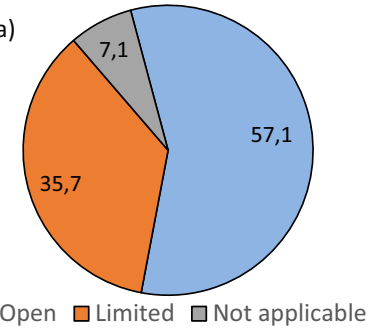

Fig. 5 a Classification of the NNCs in terms of their limits to disseminate knowledge among other stakeholders. b Percentages of NNC use of different means for knowledge dissemination and

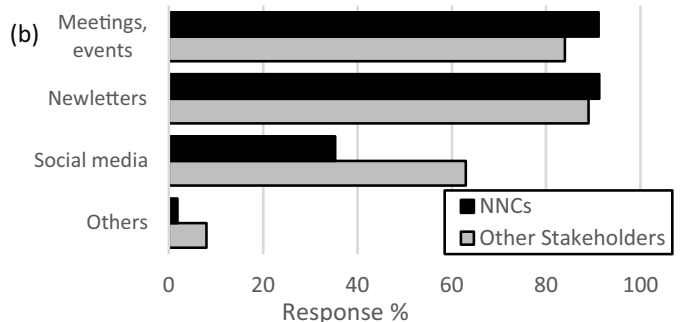

their comparison with the preferred means through which other stakeholders gather their knowledge 
NNCs, approx. $36 \%$ prefer to keep their knowledge either limited to them or their network and approx. 7\% consider themselves as not applicable in this context.

In the context of organizing a conference or a similar event, although it depends on the targeted audience, both NNCs and other stakeholders converge towards the opinion (Fig. 6) that toxicology (or human health) is generally the most attractive topic, followed by occupational safety, regulations, and public safety. In fact, the majority of the stakeholders (other than NNCs) express their foremost concern to be about human health risks which encompass (i) clear, validated, and standardized methods with guidelines for the characterization of hazardous nanomaterials and (ii) improved methods for sampling nanomaterials exposure and monitoring emissions from different processes which include the development of safe limits for occupational, consumer, or environmental exposure. A fraction of stakeholders (31\% of the NNCs and $14 \%$ of other stakeholders) additionally indicated other fields such as nanoinformatics, psychology, data sharing, and data repository to be also attractive for such events.

In the context of newsletters, stakeholders (other than NNCs) prefer information to be rather on a wide spectrum of nanosafety including information on regulatory developments, technological developments in nanosafety, nanotechnology and products, upcoming relevant events (agenda), insight on how the industrial sector is dealing with nanomaterials, and stakeholders' opinions.

\section{Network}

Most of the NNCs provide their services through a network of partners which primarily comprise research institutes and university laboratories. As shown in Fig. 7a, approx. $53 \%$ of the NNCs operate with more than 20 partners in their network, approx. 14\% have partners number between 10 and 20, approx. 28\% have 5-10 partners, and the remaining 5\% have no partners.
Depending on their objectives and service provisions, the network partners may have either specific or diverse areas of expertise, and the latter is preferred by $83 \%$ of the NNCs (Fig. 7b). In Fig. 7c, approx. $42 \%$ of NNC networks are free to join and $25 \%$ of the networks have a paid membership fee. The rest do not find the concept of network membership fee applicable to their respective situations.

Figure 8 shows that the most commonly available expertise in these networks is human toxicology which approx. $67 \%$ of the NNCs working in a network prefer to have, followed by exposure or release assessment (approx. 50\%), ecotoxicology (approx. 42\%), risk management (approx. 42\%), material science and production (approx. 42\%), etc. The areas classified as Others in Fig. 8 include legislation, chamber of commerce, hazardous waste disposal, psychology, data management, nanoinformatics, sensor technology, glass manufacturing, plasma technology, civil engineering, and semiconductors and are available in less than $10 \%$ of the NNC networks.

Around $86 \%$ of the NNCs working in a network consider likely to experience difficulties when collaborating or partnering with similar centers in other EU member states. Similar difficulties are encountered when the service provider is in a different member state than the receiver of the service. The reason can be different legislations and service costs (northern EU member states tend to have higher personnel costs than southern EU member states) to a large extent and demographic differences like different culture, language, and communication ways to a smaller extent. Consequently, these NNCs prefer to have network partners in the same EU member state to which they belong, i.e., national level network and keep their official language of correspondence to be country-specific. The remaining $14 \%$ have expanded their network to the European level or even the international level. The establishment of EC4SafeNano will ensure that these country-specific
Fig. 6 Classification of the different nanosafety-relevant topics in the order of their attractiveness for the audience in various conferences or similar events

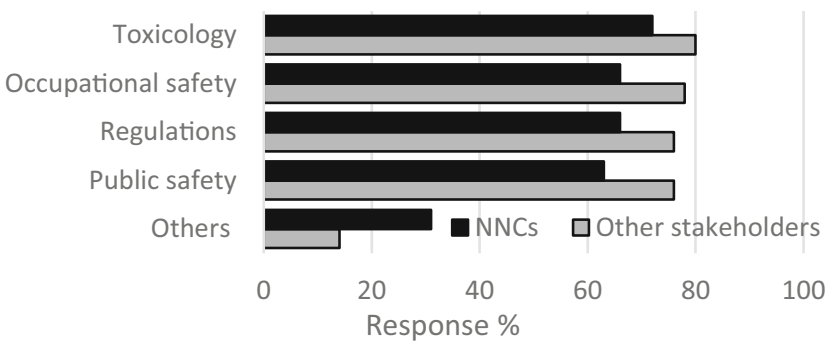



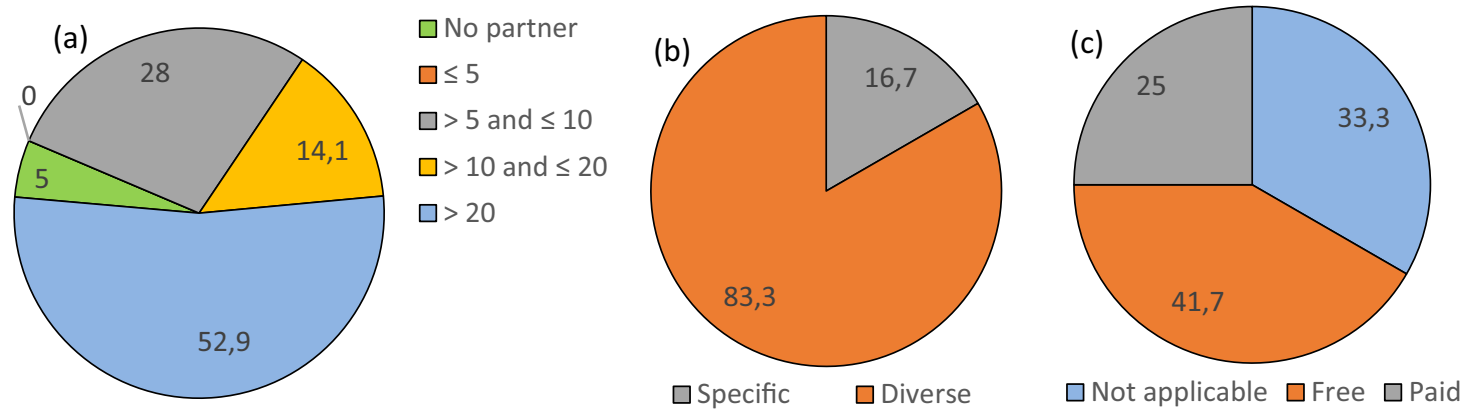

Fig. 7 a Numbers of network partners of the NNCs in percentages. b Percentages of the NNCs with network partners having either a specific or diverse nanosafety-relevant expertise. (c) Classification of NNCs on the basis of their network membership fee (free or paid)

difficulties are addressed in a harmonized way - an open collaborative network of EC4SAfeNano among NNCs and their clientele would stretch among different EU member states - making it possible to overcome localization (legislation and other) barriers. It would consequently expand the clientele of NNCs too. The inclusion of new nanomaterial-specific regulations in REACH dossiers adds an incentive for NNCs to build up and be a part of such a network. In addition, EC4SafeNano advocates NNCs to develop harmonized standard operation procedures for providing different nanosafetyrelated services which can anticipate current international differences and delays in legislations too.

Figure 9a shows that all NNCs primarily choose conferences and other similar events to develop their respective networks. Approx. 64\% of NNCs also use their personal contacts and various social events for networking. Additional preferred tools for networking include business contacts (used by approx. $57 \%$ ), meetings of industry or trade associations or societies, such as the Chemical Industries Association, the European Chemical Industry Council, the Nanotechnology Industries Association, and the British Occupational Hygiene Society (used by approx. 43\%) and online networking, such as with ResearchGate, Academia, Research Connection, and Mendeley (used by approx. 14\%). Within their respective networks, approx. $61 \%$ of the NNCs (Fig. 9b) prefer an open communication in their country-specific language. Regular face-to-face meetings are favored by approx. $69 \%$ of the NNCs for their intra-network communications, as shown in Fig. 9c. Approx. 61\% favor these regular meetings to be remote or online. Approx. 54\% of the NNCs circulate various kinds of publications (e.g., newsletters, whitepapers, internet articles, peer reviewed journal articles, etc.) within their networks to update their network partners. Social media tools like LinkedIn, Twitter, etc. are also used by approx. $46 \%$ of the NNCs for this purpose. Less preferred options include regular workshops and seminars. Looking closer on the usage of social media, Fig. $9 \mathrm{~d}$ shows that half of their users use or apply them on a general level while communicating with

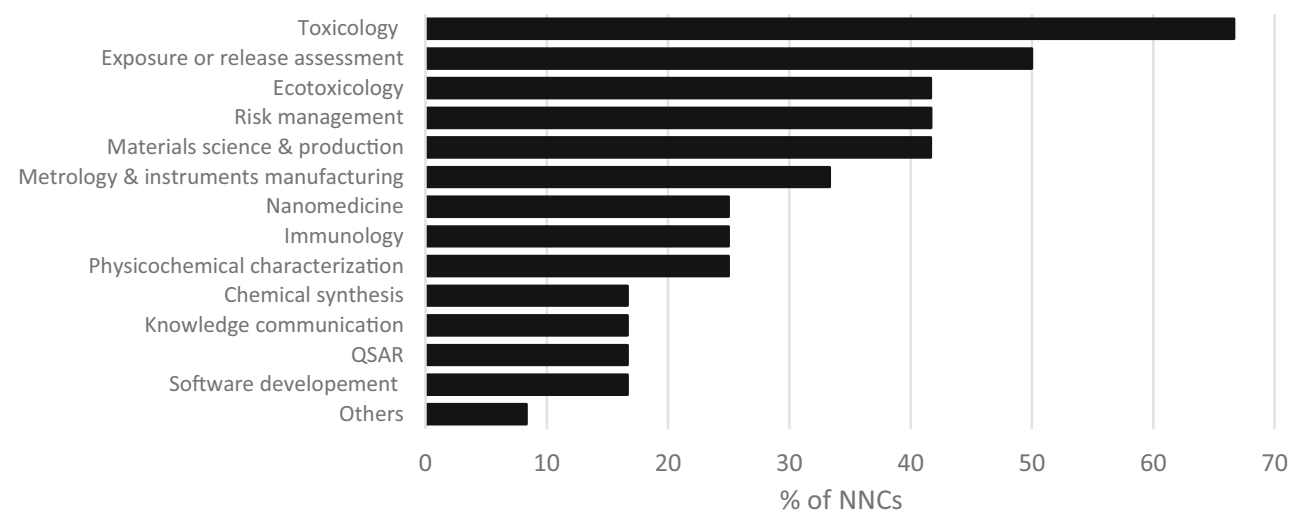

Fig. 8 Percentage of various types of nanosafety-relevant expertise in NNC networks 


\section{(a)}

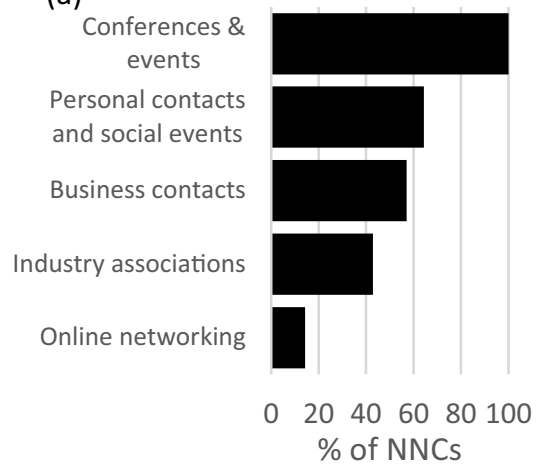

(b)

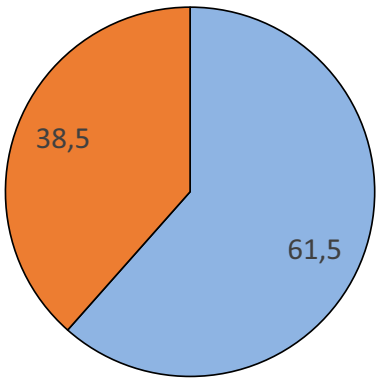

$\square$ Open (c)

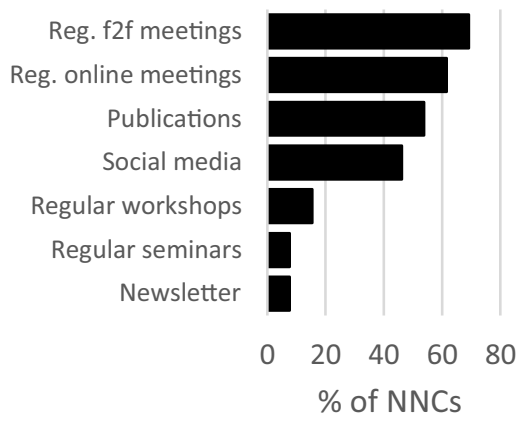

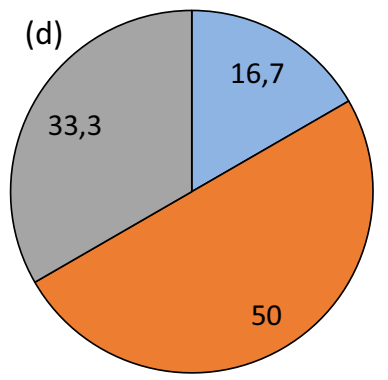

$\square$ Rare $\square$ General $\square$ Extensive

Fig. 9 a Percentages of NNC use of various means to develop their network. b Classification of NNCs in terms of their limits to disseminate knowledge within their respective networks. c Different means of intra-network communication and percentages of

network partners, $33 \%$ use them extensively, and $17 \%$ use them rarely. As Fig. 9e shows, more than half of the NNCs $(58 \%)$ choose to consult their advisory board to oversee activities within their network operations. The members of the advisory board can be either the representatives from the network partners or representatives from different stakeholder groups which are relevant to the network operations or combination of both.

\section{Clientele}

The NNCs have indicated that most of the demands on nanosafety-related services come from industries, particularly small and medium-sized enterprises (SMEs). Therefore, around $86 \%$ of the NNCs have industry as one of their clients (Fig. 10a). This is followed by the government ministries (including EU institutions) which approx. $71 \%$ of the NNCs deal with. Various non-governmental organizations (NGOs) and general public or social organizations

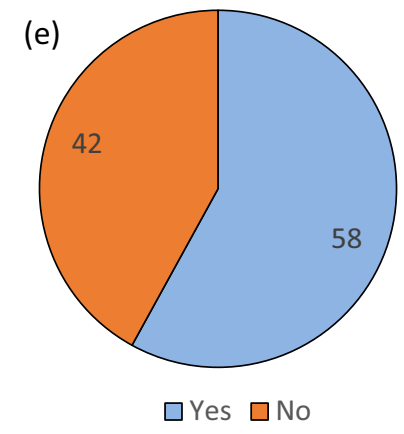

NNCs using them. d Classification of NNCs in terms of the usage limits of social media while communicating within their respective networks. e Percentages of NNCs with an advisory board for their respective networks

also form a part of the clientele in approx. 64 and $50 \%$ of the NNCs, respectively. The universities and research institutions also appear as clients of these NNCs but to a limited extent. As shown in Fig. 10b, the clients tend to avail the nanosafety-relevant services by contacting either the central office or helpdesk of the particular NNC (possible for approx. $38 \%$ of the NNCs) or any of the individual network partners (possible for approx. 38\% of the NNCs) through its website. Subscribing to the newsletters is another way for the clients to avail the services of approx. $15 \%$ of the NNCs which are mainly involved in support services. A small fraction of the NNCs (approx. 8\%) have indicated that such an access of clients is not possible in their cases for reasons of non-disclosure. During the workshop, other stakeholders also indicated that the use of the website and helpdesk phone number remains to be their prime means of access to the NNC services. Approx. $77 \%$ of the NNCs mostly communicate online with their clients, approx. $69 \%$ 

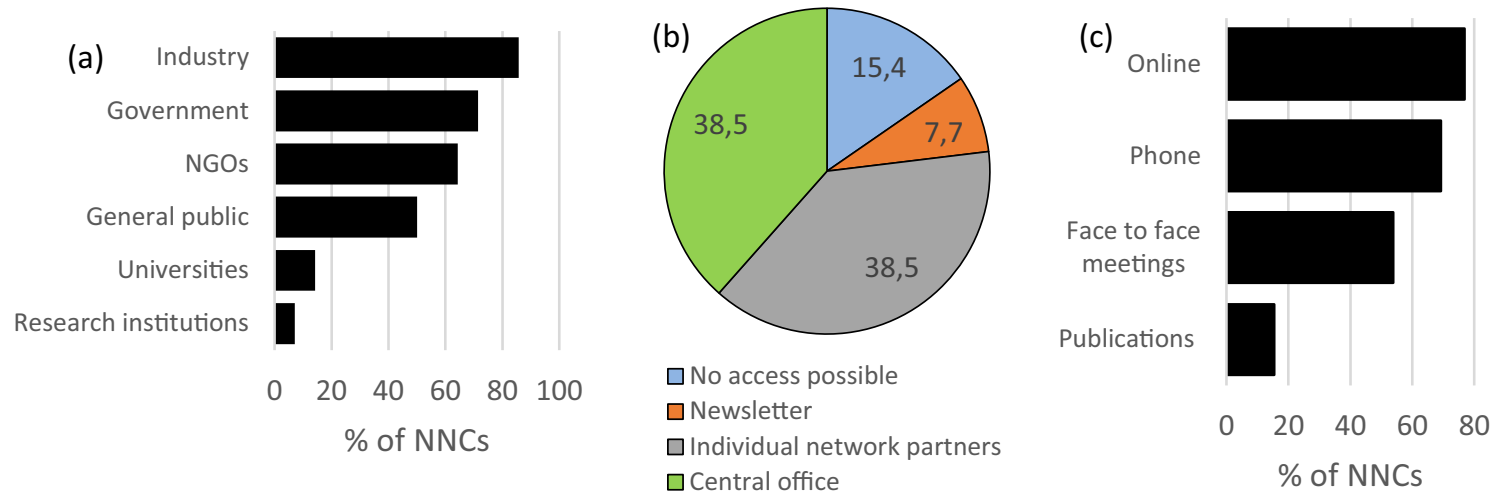

Fig. 10 a Percentages of the NNCs concerning their different clients. b Classification of means through which clients access or avail the NNC services. $\mathbf{c}$ Percentage of NNCs having different means of communication with clients

do it on the phone, approx. 54\% through face-toface meetings, and approx. 15\% through various publications, e.g., newsletters (Fig. 10c). The latter mainly comprise NNCs providing support services.

\section{Guidelines for NNC Development and Sustainability}

From the findings of this study on the operational model elements which are practiced most by the existing NNCs and nanosafety-related demands which are considered to be most vital by various stakeholders, we can propose a set of guidelines for the development and sustainability of new NNCs. These guidelines are based on the most voted options mentioned in section 3. They are described below and illustrated in Fig. 11 as a quick reference tool for a new $\mathrm{NNC}$ so that it can use this information to cut down on certain efforts which have already been undertaken by the existing NNCs on what works and what does not work to develop and operate an NNC.

1. Organization structure and funding: Based on the common practice of existing NNCs, a new NNC is suggested to adopt a non-profit type flat organization structure and should principally function on public funding as shown in Fig. 11. Its sustainable functioning can be ensured by keeping the personnel in different administrative or governance roles even at minimum level but sufficient number (or more focus) for scientific research roles. It rests upon the discretion of an NNC whether it involves personnel in all or selected roles.

2. Service provision: The services supplied by an NNC and its network should match the demand by the concerned stakeholders. Existing NNCs provide service(s) through a network (Fig. 11) including (i) publication of newsletters as support services, (ii) general training on EHS aspects, (iii) testing and analysis of physiochemical properties of ENMs and their toxicology, (iv) consultancy in risk analysis and assessment of ENMs with the development of methods and tools, and (v) conformity assessment and certification. It was observed that the existing NNCs fall back in terms of conformity assessment and certification services, compared to the demand identified here. It is also recommended that new NNCs recognize the general concern of all stakeholders for human health risks, as well as the interest of civil society in understanding and prevention of possible risks of ENMs through support services. In addition, it should also apply a market analysis to investigate if or how much the clients are willing to pay for these vital services before they invest on delivering them.

3. Objectives: To remain relevant and continue functioning in the contemporary realm of nanosafety, Fig. 11 shows that most NNCs aim for networking, connecting between industrial and academic worlds, managing relevant projects, disseminating and managing knowledge, supporting innovative research, providing access to the relevant expertise, collecting the needs in risk assessment, and/or strengthening public engagement.

4. Knowledge dissemination: To tackle ethical and social concerns surrounding ENMs, an NNC (or its network) is suggested to preferably disseminate knowledge among other stakeholders by organizing conferences and other similar events as well as by publishing recurring newsletters. For a conference or a similar event, the topics on toxicology/health, 


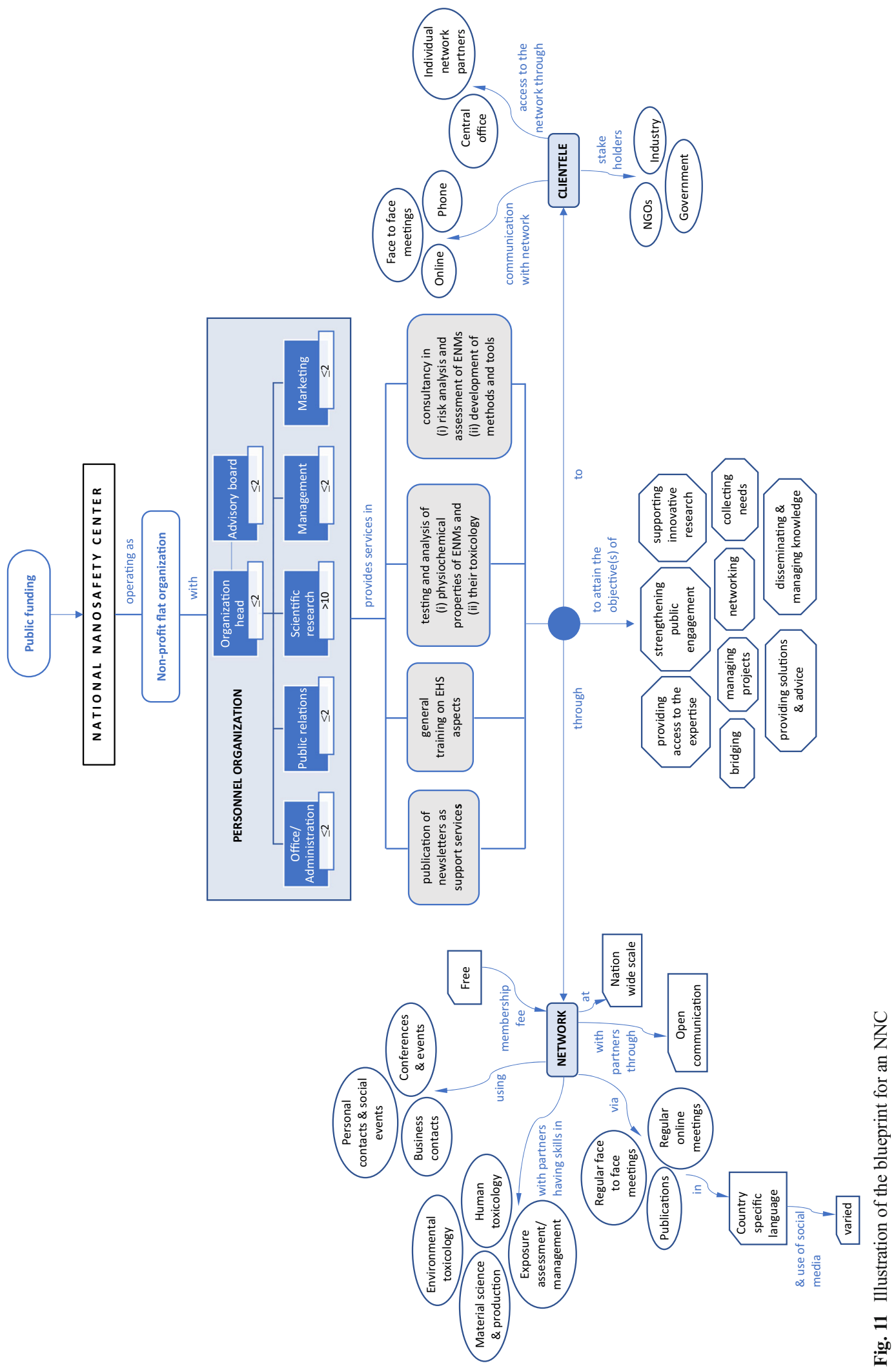


regulations, and occupational and public safety are the most engaging on a larger scale. In a newsletter, the stakeholders prefer to read information on a wide spectrum of nanosafety consisting of regulatory developments, technological developments in nanosafety, nanotechnology and products, upcoming relevant events (agenda), and insight on how the industrial sector is dealing with nanomaterials and stakeholders' opinions.

5. Network: As illustrated in Fig. 11, to provide its services to its clients, an NNC is recommended to use a network with nationwide partners who preferably have diverse ENMs relevant expertise in toxicology, exposure assessment, risk management, ecotoxicology, or material science and production. The network membership can be free to allow expansion and the NNC can attract new members through conferences, personal or business contacts, and social events. Within this network, an open communication in a country-specific language is preferable through regular face-to-face or online meetings and publications. The extent of the use of the social media in the intra-network communication and presence of an advisory board overseeing the network operations are upon the discretion of network partners.

6. Clientele: An NNC (or its network) is recommended to aim for industry and/or government and/or NGOs to have them as its clients as shown in Fig. 11. The first mode of access from a client is through the website of either the NNC (i.e., through a central office) or individual network partner. While accessing and communicating, the most efficient communication means in existing NNCs are via online meetings and/or on phone and/or face-toface meetings.

7. Connection with EC4SafeNano: An NNC is recommended to establish its connections with a European hub, like the soon-to-be-created EC4SafeNano, to enable interactions and networking with other NNCs on a European level. As per the current draft of business and governance model of EC4SafeNano [22], an NNC can join EC4SafeNano as an ordinary member or as a contributor. While an ordinary member pays a membership fee and has the right to vote for a steering committee of the hub, a contributor acts as a consultant to the hub with no right to vote. In any case, the connection of an NNC with
EC4SafeNano will not only help ensure the sustainability and relevance of this NNC but also help create an open, transparent, and trusted platform for the sharing of information, including the development of harmonized best practices and tools on national and European levels.

\section{Conclusions}

The present study recognizes the importance of establishing Nanosafety Centers on national level and proposes a blueprint for the development and operation of new NNC candidates in the EU. These centers can offer a broad range of services on the safe production, handling, and disposal of ENMs or nano-enabled products while strengthening the communication and cooperation between consumers, industry, research organizations and governments. They ensure knowledge exchange and can address regulatory, industrial and social needs related to nanosafety at least at national (and possibly at European) level.

The proposed blueprint comprises a set of guidelines and a quick reference tool based on the elements which are considered most vital for the sustainability of the NNCs by existing NNCs in Europe and other concerned stakeholders. These elements include: organization structure and funding, service provision, objectives, knowledge dissemination, networking, and clientele. The scope of the present work was to collect, analyze, and present the distilled knowledge from the efforts undertaken by the existing NNCs so far with respect to these elements. Longer term explorations of these elements and the guidelines and in situ learning are necessary. Furthermore, with respect to a Europe-wide network of NNCs the establishment of a European hub, like EC4SafeNano, is recommendable, also in order to enable and harmonize interactions and networking among NNCs on the European level.

Acknowledgments We thank all our colleagues who have responded to the online survey and provided their input during the workshop in Grenoble.

Funding Information The work carried out in this article was supported by the funding from the EU Horizon 2020 Research and Innovation Programme for the EC4SafeNano project under the Grant Agreement No 723623. 
Open Access This article is licensed under a Creative Commons Attribution 4.0 International License, which permits use, sharing, adaptation, distribution and reproduction in any medium or format, as long as you give appropriate credit to the original author(s) and the source, provide a link to the Creative Commons licence, and indicate if changes were made. The images or other third party material in this article are included in the article's Creative Commons licence, unless indicated otherwise in a credit line to the material. If material is not included in the article's Creative Commons licence and your intended use is not permitted by statutory regulation or exceeds the permitted use, you will need to obtain permission directly from the copyright holder. To view a copy of this licence, visit http://creativecommons.org/licenses/by/4.0/.

\section{References}

1. Savolainen K, Backman U, Brouwer D, Fadeel B, Fernandes T, Kuhlbusch T, Landsiedel R, Lynch I, Pylkkänen L (2013) Nanosafety in Europe 2015-2025: towards safe and sustainable nanomaterials and nanotechnology innovations. Finnish Institute of Occupational Health, Helsinki

2. Rauscher H, Rasmussen K, Sokull-Kluttgen B (2017) Regulatory aspects of nanomaterials in the EU. Chem Ing Tech 89:224-231

3. Song CH, Elvers D, Leker J (2017) Anticipation of converging technology areas - a refined approach for the identification of attractive fields of innovation. Technol Forecast Soc Change 116:98-115

4. Falk A, Schimpel C, Haase A et al (2016) Research roadmap for nanosafety part III: closer to the market (CTTM). URL: http://www.nanosafetycluster.eu/uploads/files/pdf/CTTM NSC_Roadmap_final_for_NSC.PDF. Accessed on 11 Sep 2019

5. Schulte PA, Geraci CL, Murashov V et al (2014) Occupational safety and health criteria for responsible development of nanotechnology. J Nanopart Res 16:2153

6. Fischman M, Murashov V, Borak J, Seward J (2019) Nanotechnology and health. J Occup Environ Med 61:9598

7. Liu S, Lu Y, Chen W (2018) Bridge knowledge gaps in environmental health and safety for sustainable development of nano-industries. Nano Today 23:11-15

8. Díaz-Soler BM, Martínez-Aires MD, López-Alonso M (2019) Potential risks posed by the use of nano-enabled products: a perspective from coordinators for safety and health matters. J Clean Prod 220:33-44

9. Schaper-Rinkel P (2013) The role of future-oriented technology analysis in the governance of emerging technologies: the example of nanotechnology. Technol Forecast Soc Change 80:444-452
10. Gupta N, Fischer ARH, Frewer LJ (2015) Ethics, risk and benefits associated with different applications of nanotechnology: a comparison of expert and consumer perceptions of drivers of societal acceptance. NanoEthics 9:93-108

11. Fadel TR, Steevens JA, Thomas TA, Linkov I (2015) The challenges of nanotechnology risk management. Nano Today 10:6-10

12. Karjalainen T, Hoeveler A, Draghia-Akli R (2017) European Union research in support of environment and health: building scientific evidence base for policy. Environ Int 103:5160

13. Bopp SK, Barouki R, Brack W, Dalla Costa S, Dorne JCM, Drakvik PE, Faust M, Karjalainen TK, Kephalopoulos S, van Klaveren J, Kolossa-Gehring M, Kortenkamp A, Lebret E, Lettieri T, Nørager S, Rüegg J, Tarazona JV, Trier X, van de Water B, van Gils J, Bergman Å (2018) Current EU research activities on combined exposure to multiple chemicals. Environ Int 120:544-562

14. Hodson L, Geraci C, Schulte P (2019) Continuing to protect the nanotechnology workforce: NIOSH nanotechnology research plan for 2018-2025. NIOSH publication 2019-116. https://doi.org/10.26616/NIOSHPUB2019116

15. Hansen SF, Broomfield M, Pelsy F (2016) Support for 3rd regulatory review on nanomaterials - environmental legislation (Report for European Commission DG Environment). https://doi.org/10.2779/49879. https://publications.europa. eu/en/publication-detail/-/publication/cf73479b-b601-11e69e3c-01aa75ed71a1/language-en. Accessed on 11 Sep 2019)

16. European Centre for Risk Management and Safe Innovation in Nanomaterials \& Nanotechnologies (EC4SafeNano). http://ec4safenano.eu/. Accessed on 11 Sep 2019

17. EC4SafeNano Workshop: Blueprint for NanoSafety Platform Development \& Sustainability (BNP-DS). https://www.nanosafetycluster.eu/news/279/66/EC4 SafeNano-Workshop-Blueprint-for-NanoSafety-PlatformDevelopment-Sustainability.html. Accessed on 11 Sep 2019

18. George G, Bock AJ (2011) The business model in practice and its implications for entrepreneurship research. Entrepreneurship Theory Practice 35:83-111

19. Berglund H, Sandström C (2013) Business model innovation from an open systems perspective: structural challenges and managerial solutions. Int J Prod Dev 18:274-285

20. Munsch K (2009) Open model innovation. Res Technol Manag 52:48-52

21. ECHA (2019) REACH Guidance for nanomaterials, URL: https://echa.europa.eu/nl/-/get-ready-for-new-reachrequirements-for-nanomaterials. Accessed on 27 Jan 2020

22. EC4SafeNano D3.2 and D5.2. http://ec4safenano.eu/results. Accessed on 27 Jan 2020

Publisher's Note Springer Nature remains neutral with regard to jurisdictional claims in published maps and institutional affiliations. 